\title{
Frequency-Domain Simulation and Analysis of Vehicle Ride Comfort based on Virtual Proving Ground
}

\author{
Jie Gao ${ }^{1,2}$, Ke Chen $^{1 *}$ \\ ${ }^{1}$ School of Automobile and Transportation, Shenyang Ligong University, Shenyang 110159, Liaoning China \\ ${ }^{2}$ Dalian Port traffic Vocational and Technical School, Dalian 116013, Liaoning China \\ * Corresponding author's Email: chen_ke@163.com
}

\begin{abstract}
Vehicle ride comfort is one of the important properties of modern car. This paper takes a practical model for reference section and a virtual model is established based on virtual proving ground (VPG). By calculating and analyzing simulation results, we can comprehensively and accurately evaluate the model, and do further study of different factors impacting on ride comfort. The main researches include: the multi-body dynamics model and random road surface are established based on VPG. The body acceleration time-domain curves and frequency-domain power spectrums are obtained by LS-DYNA. Total weighted acceleration of vehicle ride comfort is evaluated by root-meansquare method. The effects of suspension system, tire pressure and speed on the vehicle ride comfort are analyzed. Simulation results show that the simulation of vehicle ride comfort based on VPG can truly reflect real test condition and the results achieved are accurate and reliable.
\end{abstract}

Keywords: vehicle; ride comfort; suspension; tire pressure; power spectral density; Virtual Proving Ground (VPG)

\section{Introduction}

For the vehicle ride comfort studies, experimental research and theoretical analysis method are generally used to simulate vehicle ride comfort. The test research method on real vehicle test uses "design-trial production-test improvement-further trial production" process to simulate vehicle ride comfort. This method is greatly influenced by objective factors and the test results feed back slowly, needing high cost and long cycle. The theoretical analysis method simplifies real vehicle model result in many important characteristics of vehicle that can not be accurately analyzed. Therefore, the analysis results have great errors and low accuracy. In order to avoid the limitations of test research and theoretical analysis methods, with the development of computer simulation technology, new automotive test method based on virtual simulation technology emerged $[1,2]$. Virtual vehicle models are able to simulate various response characteristics and adjust model design parameters visually and timely. Moreover, simulation results are comprehensive and accurate.

At present, ADAMS is widely used on common simulation application $[3,4]$, but the accuracy is limited for the rigid body vehicle model. Considering characteristics of flexible body, the simulation is difficult and the simulation results are not accurate enough by ADAMS. The virtual proving ground (VPG) $[5,6]$ approach uses a nonlinear dynamic finite element code (LS-DYNA 3D) which expands the application boundary outside the classic linear static assumptions. The whole vehicle model is established by VPG. The model consists of the front and rear suspensions, tires and vehicle body. The VPG approach also uses realistic boundary conditions of tire/road surface interactions. In short, VPG enables a more reasonable, fast and ac- 
curate study for vehicle ride comfort.

In this paper, a multi-body dynamics simulation virtual vehicle model and a random road surface model are established based on VPG. LS-DYNA is used to solve simulation results. This method of studying vehicle ride comfort realized on computer has important practical significance for improving vehicle ride comfort, reducing production costs and shorting development cycle. And the feasibility of the integrated CAE analysis methodology is verified.

\section{Simulation Model}

In this paper, according to the actual vehicle, a multibody dynamics simulation virtual vehicle model and a random road surface model are established based on VPG.

The main parameters of the actual vehicle and the simulation model studied in this paper are shown in Table 1 .

Table 1 Main parameters of vehicle

\begin{tabular}{|c|c|c|c|}
\hline \multicolumn{2}{|c|}{ Details } & $\begin{array}{l}\text { Actual } \\
\text { vehicle }\end{array}$ & $\begin{array}{c}\text { Simulation } \\
\text { model }\end{array}$ \\
\hline \multicolumn{2}{|c|}{ Vehicle quality(Kg) } & 1354 & 1333 \\
\hline \multicolumn{2}{|c|}{ Engine type } & $2.0 \mathrm{~L} \mathrm{I} 4$ & $2.0 \mathrm{~L} \mathrm{I} 4$ \\
\hline \multicolumn{2}{|c|}{ Tire Size } & P185/65R14 & P195/65R15 \\
\hline \multirow{2}{*}{$\operatorname{Track}(\mathrm{mm})$} & Front & 660 & 735 \\
\hline & Rear & 676 & 740 \\
\hline \multicolumn{2}{|c|}{ Wheelbase (mm) } & 2642 & 2600 \\
\hline
\end{tabular}

\subsection{Vehicle body finite element model}

According to the actual vehicle body geometry, the vehicle body finite element model is established. The white body of the CAD model is converted into a CAE model based on Pre-processing of VPG.

The Body-in-white is defined as shell unit. The thickness of steel plate is defined according to actual vehicle body thickness and set by defining the thickness of four node quadrilateral elements. The vehicle body is meshed by topology technology.

The materials of body-in-white uses primarily lowcarbon steel sheet to draw better performance. In this paper, we mainly use 20 rigid materials in VPG material library where material density is $7.83 \times 10^{-6} \mathrm{Kg} /-$ $\mathrm{mm}^{3}$, elastic modulus is $2.07 \times 10^{5} \mathrm{MPa}$ and poisson ratio is 0.28 .

Connect various components of the body using * Constrained_Rigid Bodies on VPG. The advantage of it is that different rigid bodies become into a single rigid body and the freedom of vehicle body decreases to six degree which shortens the computation time.
The vehicle multi-body finite element model of the test is shown in Figure 1 which has totally 95394 units and 98390 nodes.

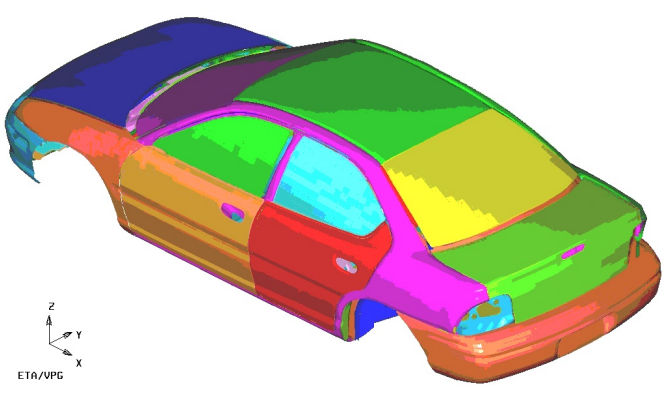

Figure 1 Vehicle body finite element model

\subsection{Suspension model}

The suspensions used on vehicle are McPherson strut suspension. The suspension element type and component materials attribute are shown in Table 2 where material density is $8.73 \times 10^{-9}$ ton $/ \mathrm{mm}^{3}$, elastic modulus is $2.07 \times 10^{5} \mathrm{MPa}$ and Poisson ratio is 0.28 .

Table 2 Definition of suspension element type and component materials attribute

\begin{tabular}{|c|c|c|}
\hline Part Name & Element Type & $\begin{array}{c}\text { Material } \\
\text { Attribute }\end{array}$ \\
\hline stabilizer bar & beam element & flexibility \\
\hline flexible bush & $\begin{array}{c}\text { Six zero-length discrete } \\
\text { springs (six translations, three } \\
\text { rotations), and six zero-length } \\
\text { discrete damping units (three } \\
\text { translations, three rotations) }\end{array}$ & $\begin{array}{c}\text { discrete } \\
\text { non-linear } \\
\text { flexible }\end{array}$ \\
\hline $\begin{array}{c}\text { suspension } \\
\text { spring }\end{array}$ & spring element & $\begin{array}{c}\text { non-linear } \\
\text { flexible }\end{array}$ \\
\hline $\begin{array}{c}\text { suspension } \\
\text { shock absorber } \\
\text { damper }\end{array}$ & damping unit & $\begin{array}{c}\text { non-linear } \\
\text { viscous }\end{array}$ \\
\hline $\begin{array}{c}\text { swing arm and } \\
\text { pull rod }\end{array}$ & beam element & rigid \\
\hline $\begin{array}{c}\text { strut body and } \\
\text { piston rod }\end{array}$ & beam element & flexible \\
\hline $\begin{array}{c}\text { vibration and } \\
\text { rebound lock }\end{array}$ & $\begin{array}{c}\text { non-linear spring element } \\
\text { modeling, non-linear spring } \\
\text { stifness were only defined in } \\
\text { suspension vibration and } \\
\text { rebound movement }\end{array}$ & non-linear \\
flexible \\
\hline $\begin{array}{c}\text { steering } \\
\text { knuckle }\end{array}$ & beam element & rigid \\
\hline under corbel & beam element & rigid \\
\hline others & beament & rid \\
\hline
\end{tabular}

Define constraints between suspension parts:

- the under corbel and one end of the sleeve uses rigid connections;

- the strut body and piston rod uses cylindrical pair restriction conditions;

- the steering knuckle and pull rod uses spherical pair restriction conditions; 
- the under corbel of the rear suspension and shaft uses revolute joint restriction conditions.

After establishing geometric structural parameters and defining material attributes, element types and parts constraints, the front and rear suspension models are shown in Figure 2.

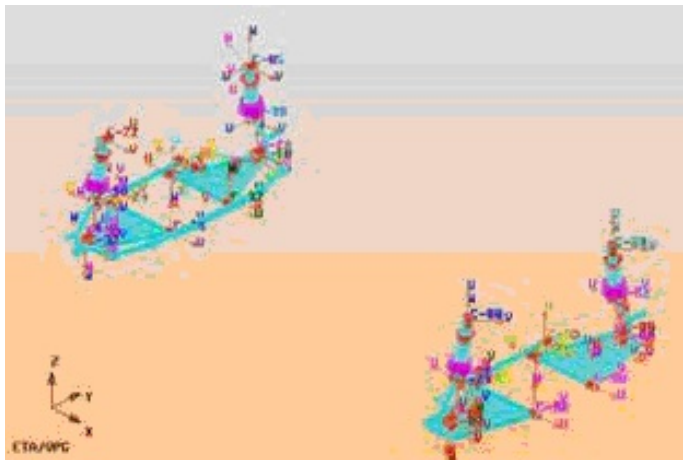

Figure 2 Front and rear suspension model

The connection between body and suspension is by a new six-part component of BODY, BODYNE, FRAME on front and rear suspension and rigid body.

There are two ways of connecting of body and suspension: ${ }^{*}$ Constrain_Rigid_Bodies way for rigid parts connection and *Constrain_Extra_Nodes way for rigid part and flexible part connection.

\subsection{Tire model}

Tire models are 195/65R15 whose specific parameters are shown in Table 3.

Table 3 Main parameters of tire

\begin{tabular}{|c|c|c|}
\hline & Item & Parameter values \\
\hline 1 & Tire section width(mm) & 195 \\
\hline 2 & Tire cross-section aspect ratio & 065 \\
\hline 3 & Rim diameter(inch) & 15 \\
\hline 4 & Rim width(inch) & 6.5 \\
\hline 5 & Tire radius(mm) & 310.75 \\
\hline 6 & Tire pressure $(\mathrm{Mpa})$ & 0.25 \\
\hline 7 & Tire quality $(\mathrm{Kg})$ & 22.5 \\
\hline
\end{tabular}

Finite element model of upper and lower tread and swathe use eight-node hexahedron solid elements, choosing super-elastic rubber material; top cord and lateral wall use elastic shell elements; wheel hub and wheel rim use rigid shell elements [7].

The total number of tire model is 2144 units, of which Shell units are 1184, Solid units are 960 and nodes are 2480 .

The tire finite element model is shown in Figure 3.

The definition of constraints between tire and suspension is rotation pair; the relative penalty stiffness

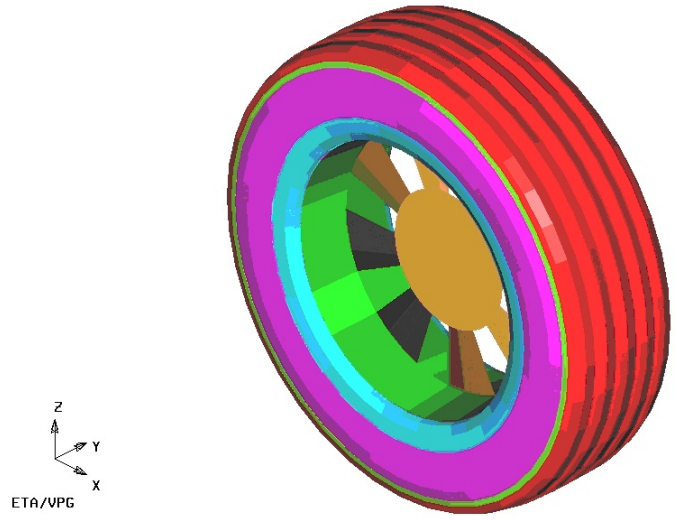

Figure 3 Tire finite element model

between wheel rim and knuckle centre spindle is 1.0 and the contact type between tire and road surface is unilateral contact [8].

\subsection{Simulation model of whole vehicle}

The total mass of suspension, tires and body is $1333 \mathrm{~kg}$. Its front axle load is $40 \%$ and rear axle load is $60 \%$. A rigid quality-point instead of other parts of the quality of the car is added to the body. Adjust the vehicle centroid position and whole vehicle model is formed, while gravity acceleration is established at the same time.

The vehicle simulation model is shown in Figure 4.

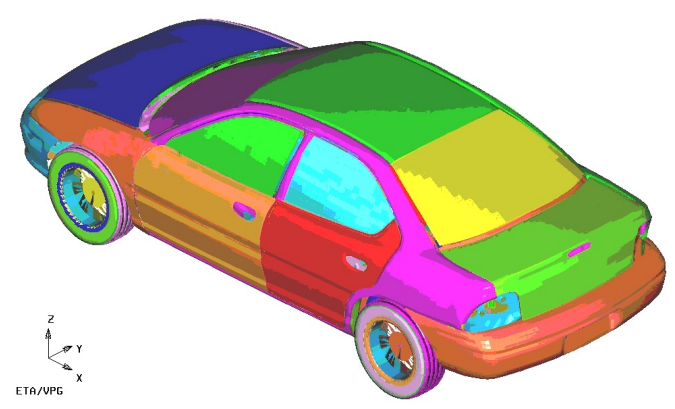

Figure 4 Vehicle simulation model

\subsection{Random road surface model}

ccording to the literature $[9,10]$, the road surface roughness power spectral density is expressed as:

$$
G_{q}(n)=G_{q}\left(n_{0}\right)\left(n / n_{0}\right)^{-\omega}
$$

where $n$ is spatial frequency; $\omega$ is frequency index, $\omega=2 ; n_{0}$ is reference spatial frequency, $n_{0}=0.1$.

In order to make convenience for calculation and time-domain signal analysis, it is usual to convert spatial frequency power spectral density $G_{q}(n)$ into time 
frequency power spectral density $G_{q}(f)$ :

$$
G_{q}(f)=v^{-1} G_{q}(n)=G_{q}\left(n_{0}\right) n_{0}^{2} v / f^{2}
$$

The road acceleration power spectral density $G_{q}(f)$ and body vertical acceleration power spectral density $G_{z}(\omega)$ are expressed as:

$$
\begin{gathered}
G_{q \&}(f)=(2 \pi f)^{2} G_{q}(f)=4 \pi^{2} G_{q}\left(n_{0}\right) n_{0}^{2} v \\
G_{q \& \&}(f)=(2 \pi f)^{4} G_{q}(f)=16 \pi^{4} G_{q}\left(n_{0}\right) n_{0}^{2} v f^{2} \\
G_{z \& \&}(\omega)=|H(j \omega)|_{z \& \& q \& \&}^{2} G_{q \& \&}(\omega)
\end{gathered}
$$

where $v$ is speed; $|H(j \omega)|_{z \& \& q \& \&}^{2}$ is acceleration amplitude frequency.

The road model established on VPG is in line with the United States MGA standard corrugated surface (Pave Surface), the equivalent of C-level gravel road in our Country. The spatial distribution of road roughness is shown in Figure 5.

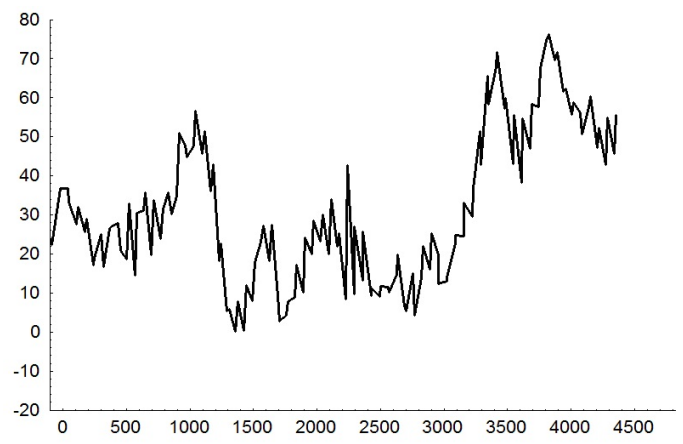

Figure 5 Spatial distribution of C-level road surface roughness

The C-level road surface roughness power spectral density is expressed as:

$$
G_{q}(n)=\left\{\begin{array}{l}
256 \times 10^{6} \mathrm{~m}^{3}, 0 \leq n \leq 0.1 \\
265 \times 10^{6}\left(n / n_{0}\right)^{2} m^{3}, 0.1 \leq n \leq 2 \\
0 \mathrm{~m}^{3}, n \leq 2
\end{array}\right.
$$

6.

The random road surface model is shown in Figure

The contact between road surface and tire is unilateral contact which is * Contact_Automatic_Nodes_To_Surface on VPG. Contact algorithm uses penalty method. At the same time, the static friction coefficient (FS) and dynamic friction coefficient (FD) values are set to 0.7.

After model building and constraints settings, the simulation system is shown in Figure 7.

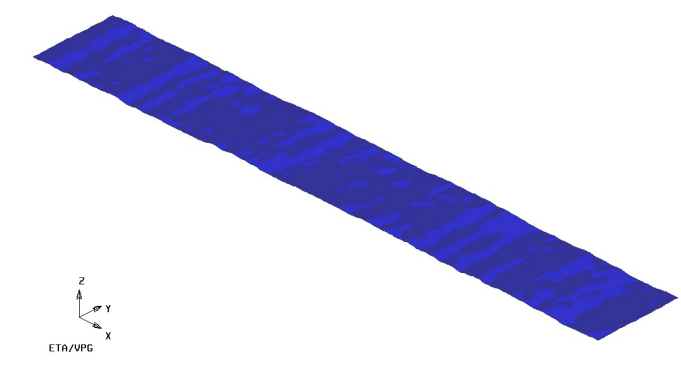

Figure 6 Random road surface model

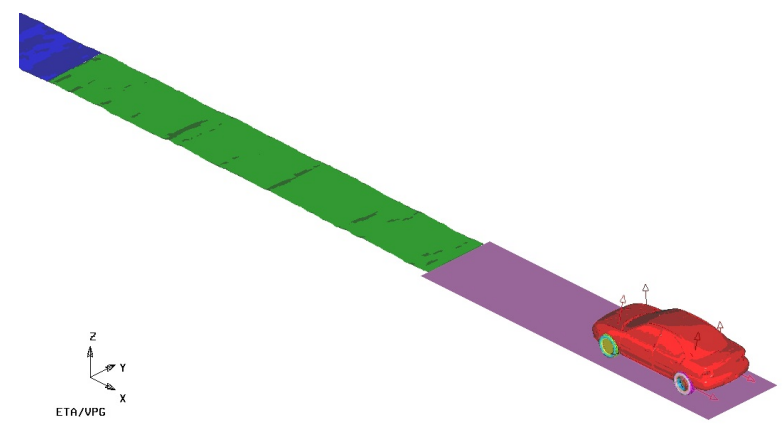

Figure 7 Simulation system

\section{Vehicle Dynamic Equation}

To predict and analyze vehicle ride comfort, it must be a reasonable vehicle dynamics model. According to the requirements of freedom and the actual use of test equipment, it is reasonable to simplify the vehicle system. In addition, because the evaluation of ride comfort analysis is mainly about Z-direction acceleration value on vehicle body, combining with the analysis of this issue, a half six degrees of freedom vehicle dynamic model will be set and made the following assumptions [11-14] will be made:

- Automotive structures symmetrical, uniform mass distribution and the road around the wheel suffer the same incentives.

- Simplify the mechanical properties of the tire for the general spring, regardless of the tire damping effect on the vehicle vibration.

- Vehicle vibration system is linear vibration system. Suspension stiffness and damping are considered constant. Simultaneously the impact of personal vehicles is negligible. The "peoplechairs-car" is simplified to a series system.

- Vehicle operating conditions is constant linear driving.

According to the above assumptions, based on random vibration theory, kinetic equation [15] of virtual model of vehicle ride comfort simulation is established 
based on multi-body system dynamics analysis method. The equation is expressed as:

$$
\begin{aligned}
{[M]\{\ddot{q}\}+[C]\{\dot{q}\} } & +[K]\{q\} \\
& =\left[K_{t}\right]\{r\}+\left[C_{t}\right]\{\dot{r}\}
\end{aligned}
$$

Where: $M$ is mass matrix of vehicle; $q$ is generalized coordinates of vehicle vibration system; $C$ is damping matrix of vehicle; $K$ is stiffness matrix of vehicle; $K_{t}$ is stiffness matrix of tire; $C_{t}$ is damping matrix of tire; $r$ is road input vector.

\section{Simulation Results and Analysis}

The main factor affecting the vehicle ride comfort is the vibration which is caused by road irregularities acting on wheel and through vehicle suspension system delivered to body. The stiffness and damping of suspension system directly affect the strength of the vehicle vibration and the vehicle ride comfort. In addition, the tire pressure and speed also have important implications on vehicle ride comfort. So in this paper the major aim is to study the effects of suspension system, tire pressure and speed on vehicle ride comfort.

Analyze and evaluate established vehicle model using time-domain and frequency-domain methods [16]. Time-domain analysis can accurately reflect vehicle degrees of freedom changes with time and solve the nonlinear problems in ride comfort study; the frequencydomain analysis can directly observe the vehicle distribution of power spectral density in a specific frequency or band frequency. In order to evaluate and analyze suspension stiffness, damping and tire pressure impact on ride comfort, according to different frequencies of human sensitivity to vibration signals and spectral density distribution in frequency range, the most sensitive frequency of the human body is avoided for the purpose of getting good ride comfort.

The specific test methods are: the body vertical acceleration and variation of power spectral density are calculated at $60 \mathrm{Km} / \mathrm{h}$ speed and $0.25 \mathrm{MPa}$ tire pressures under $\mathrm{C}$-level road by changing parameters of suspension stiffness, suspension damping, tire pressure and speed. The vehicle ride comfort is evaluated with total weighted acceleration root-mean-square value [17].

\subsection{Suspension effect on vehicle ride comfort}

\subsubsection{Suspension stiffness}

Initial stiffness of suspension is defined by stiffness curve that is shown in Figure 8.

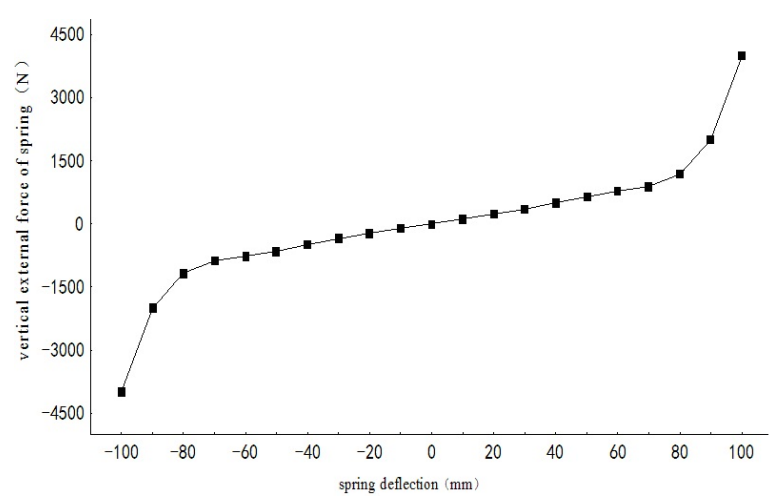

Figure 8 Initial stiffness curve of suspension

Under the other conditions remain unchanged, rigid body models of flexible coupling suspension are simulated by changing parameters of suspension stiffness at rate of $25 \%$. Compare body vertical acceleration of rigid body models of flexible coupling suspensions at different suspension stiffness, as shown in Figure 9.

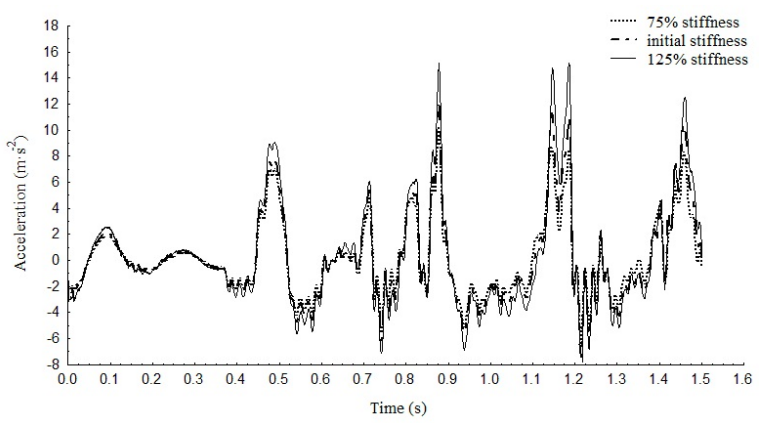

Figure 9 Compare body vertical acceleration at different suspension stiffness

As seen from Figure 9, with suspension stiffness increased, body vertical acceleration enlarges.

Time-domain signal is converted to frequency domain signal by Fourier transform. Body vertical acceleration power spectral density is obtained at different suspension stiffness. Compare body vertical acceleration power spectrum, as shown in Figure 10.

As seen from Figure 10, with suspension stiffness increased, peak of main resonance amplitude increases and peak of sub-formant amplitude decreases, but frequency of formants does not change.

Area of envelope curve is the total power of body vertical acceleration. With peak of main resonance amplitude increased, peak of sub-formant amplitude decreases, which shows simulation result meets law of conservation of energy. In addition, with stiffness increased, amplitude of body vertical acceleration power spectral density increases. So reducing suspension stiffness at reasonable limits will benefit to improve 


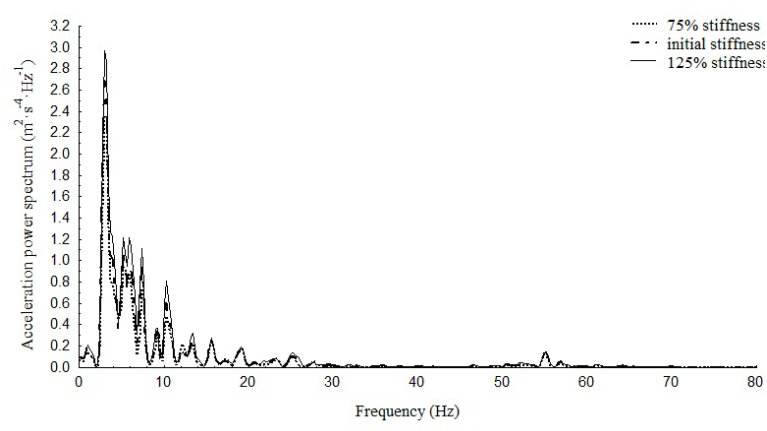

Figure 10 Compare body vertical acceleration power spectrum at different suspension stiffness

vehicle ride comfort.

\subsubsection{Suspension damping}

Initial damper of suspension is defined by the damper curve that is shown in Figure 11.

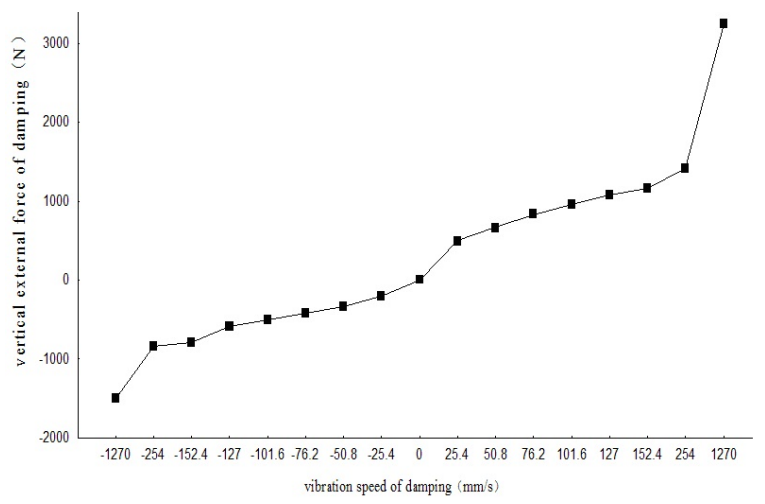

Figure 11 Initial damping curve of suspension

Under the other conditions remain unchanged, rigid body models of flexible coupling suspension are simulated by changing parameters of suspension damping at rate of $25 \%$. Compare body vertical acceleration of rigid body models of flexible coupling suspensions at different suspension damping, as shown in Figure 12.

As seen from Figure 12, with suspension damping increased, body vertical acceleration enlarges.

Time-domain signal is converted to frequency domain signal by Fourier transform. Body vertical acceleration power spectral density is obtained at different suspension damper. Compare body vertical acceleration power spectrum, as shown in Figure 13.

As seen from Figure 13, with suspension damping increased peak of main resonance amplitude decreases and peak of sub-formant amplitude small decreases, but high frequency region of body vertical acceleration power spectrum density does not change.

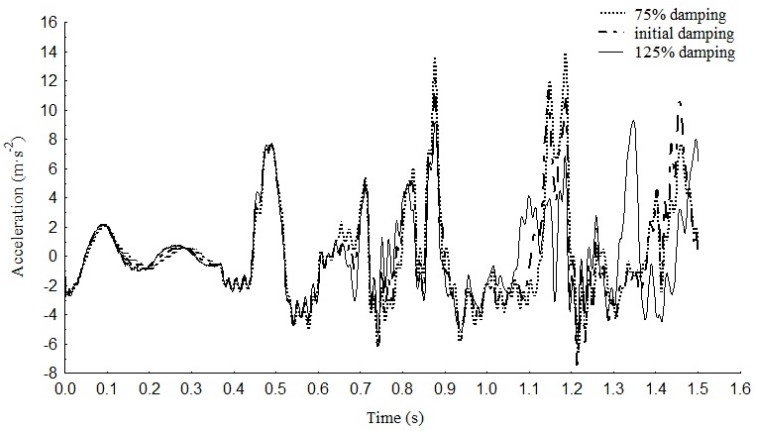

Figure 12 Compare body vertical acceleration at different suspension damping

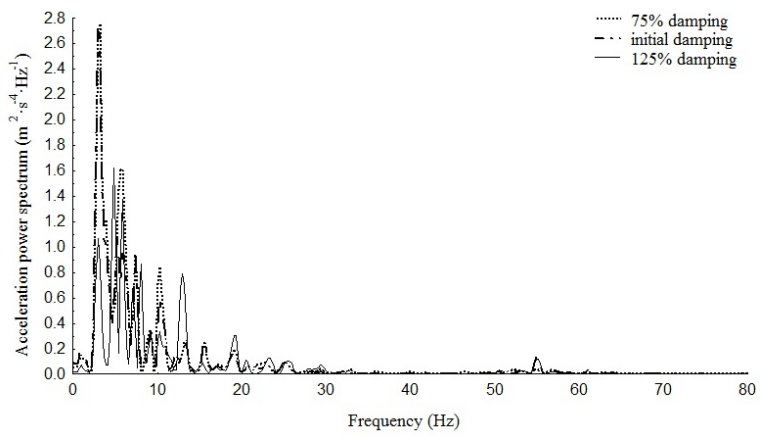

Figure 13 Compare body vertical acceleration power spectrum at different suspension damping

As analysis of suspension stiffness affecting the vehicle ride comfort, with suspension damping increased, amplitude of body vertical acceleration power spectral density decreases, which indicates suspension damping is energy-absorbing component. Increasing suspension damping is beneficial to absorbing vibration. So increasing suspension damping at reasonable limits will help to improve vehicle ride comfort.

In summary, suspension stiffness and suspension damping have direct impact on vehicle ride comfort which is consistent with the feeling of occupants. Simulation results verify that the suspension models established are reasonable and reliable.

\subsection{Tire pressure effect on vehicle ride comfort}

Under the other conditions remain unchanged, rigid body models of rigid and flexible coupling suspensions are simulated at $0.2 \mathrm{Mpa} 0.25 \mathrm{Mpa}$ and $0.3 \mathrm{Mpa}$ tire pressures.

Compare body vertical acceleration of rigid body models of flexible coupling suspensions at different tire pressures, as shown in Figure 14.

As seen from Figure 14, with tire pressure increased, body vertical acceleration enlarges.

Time-domain signal is converted to frequency do- 


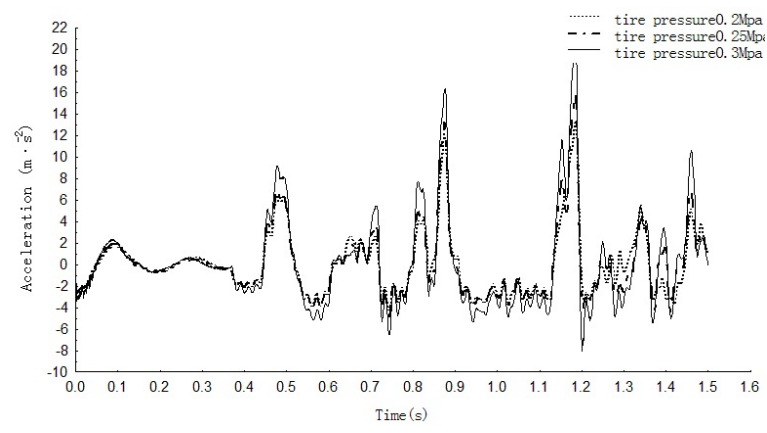

Figure 14 Compare body vertical acceleration at different tire pressures

main signal by Fourier transform. Body vertical acceleration power spectral density is obtained at different tire pressures. Compare body vertical acceleration power spectrum, as shown in Figure 15.

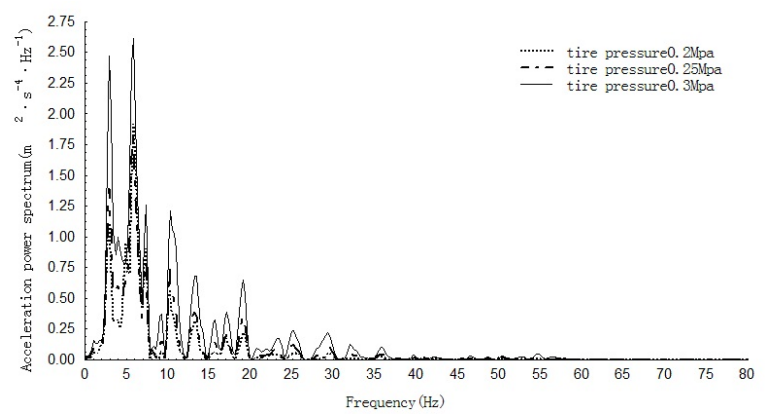

Figure 15 Compare body vertical acceleration power spectrum at different tire pressures

As seen from Figure 15, with tire pressure increased peak of main resonance amplitude decreases and peak of sub-formant amplitude small decreases, but high frequency region of body vertical acceleration power spectrum density does not change.

The simulation results show that tire pressure has impact on ride comfort. As tire pressure increases, the tire radial stiffness enlarges, buffer capacity of tire deteriorate reduces and amplitude of power spectral density increases which is consistent with the feeling of occupants. Simulation results verify that the tire model established is reasonable and reliable.

\subsection{Speed effect on vehicle ride comfort}

Under the other conditions remain unchanged, rigid body models of rigid and flexible coupling suspensions are simulated at $50 \mathrm{~km} / \mathrm{h}$ speed $60 \mathrm{~km} / \mathrm{h}$ speed and $60 \mathrm{~km} / \mathrm{h}$ speed.

Compare body vertical acceleration of rigid body models of flexible coupling suspensions at different speeds, as shown in Figure 16.

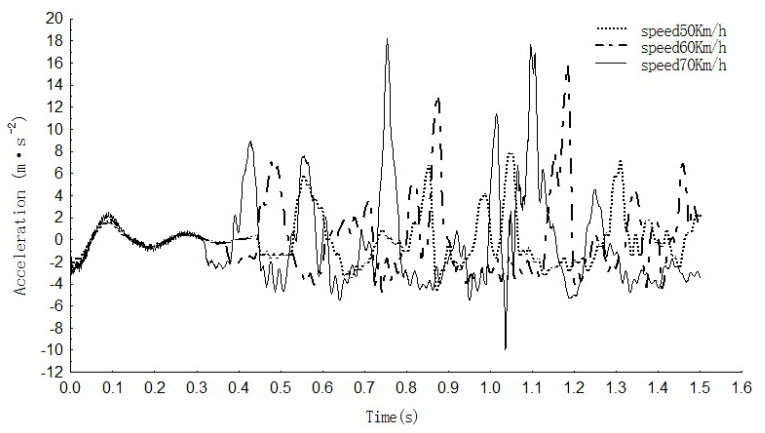

Figure 16 Compare body vertical acceleration at different speeds

As seen from Figure 16, with speed increased, body vertical acceleration enlarges.

Time-domain signal is converted to frequency domain signal by Fourier transform. Body vertical acceleration power spectral density is obtained at different speeds. Compare body vertical acceleration power spectrum, as shown in Figure 17.

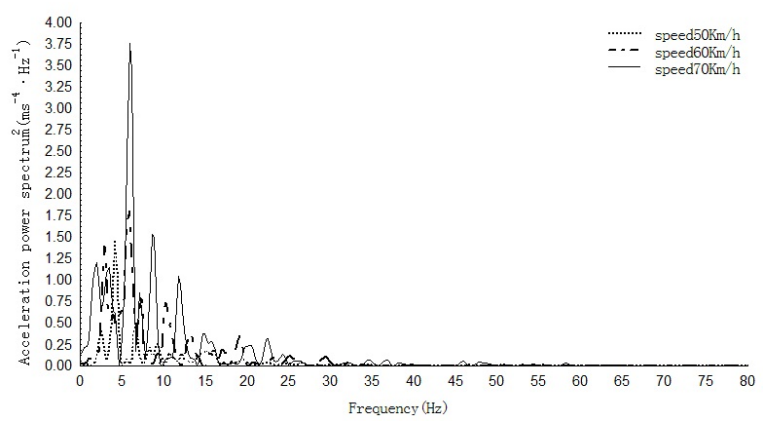

Figure 17 Compare body vertical acceleration power spectrum at different speed

As seen from Figure 17, with speed increased, peak of main resonance amplitude decreases and peak of sub-formant amplitude small decreases, but high frequency region of body vertical acceleration power spectrum density does not change.

The simulation results show that vehicle speed has impact on ride comfort. As speed increases, the body vertical acceleration and amplitude of power spectral density increases which is consistent with the feeling of occupants. Simulation results verify that the vehicle model established is reasonable and reliable.

\section{Summary}

In this paper, using the VPG Approach to simulate real road driving conditions, an integrated CAE environment was used to test vehicle's performance. The results obtained are as follows: 
1) A virtual vehicle model is established based on VPG. The calculation results are obtained by LS-DYNA. The analysis of time-domain can accurately reflect the change of vehicle preferences with time and its results can be directly compared with real vehicle test data. Moreover, it can solve non-linear problems well. The analysis of frequency-domain can directly observe distribution amplitude under a particular frequency or frequency band and can accurately analyze various parameters effect on vehicle ride comfort.

2) It is essential to choose reasonable suspension stiffness damper for vehicle ride comfort. Reducing suspension stiffness or increasing suspension damper will benefit to improve vehicle ride comfort at reasonable limits.

It is also essential to choose reasonable tire pressure for vehicle ride comfort. Reducing tire pressure within reasonable limits will benefit to improve vehicle ride comfort at reasonable limits.

Vehicle speed has impact on ride comfort. Reducing vehicle speed will benefit to improve vehicle ride comfort.

3) The VPG Approach which is presented in this study can be utilized to analyze not only rigid dynamics, but also flexible dynamics for vehicle ride comfort. In modeling process, we fully considered the flexible body and the rigid body properties; described the component materials in details, connections and constraints in details and considered non-linear properties of flexible elements and damper elements to improve the accuracy of the simulation model and truly describe the real vehicle test situation. The simulation results are accurate and reliable.

In summary, the method of studying vehicle ride comfort realized on computer has important practical significance for improving vehicle ride comfort, reducing production costs and shorting development cycle.

\section{References}

[1] Wang Guoquan, Study on Virtual Test of Vehicle Ride Comfort, China Agricultural University, Doctor of Science thesis, 2002.

[2] A. Kuznetsov, M. Mammadov, I. Sultan and E. Hajilarov, "Optimization of a quarter-car suspension model coupled with the driver biomechanical effects", Journal of Sound and Vibration, vol.330(12), pp.2937-2946, 2011.

[3] P. S. Els, N. J. Theron, P. E. Uys and M. J. Thoresson, "The ride comfort vs handling compromise for off-road vehicles", Journal of Terramechanics, vol.44(4), pp.303-317, 2007.
[4] X. Q. Zhang and B. Yang, "Simulation and Analysis of Ride Comfort under Random Road based on ADAMS", Shanghai Auto, (8), pp.18-23, 2011.

[5] Y. Zhang and A. Tang, "The CAE Revolution and the Development of the Virtual Proving Ground Approach", The 4th International LS-DYNA Conference, Minneapolis, Minnesota, 1996.

[6] D. C. Lee and C. S. Han, "CAE (computer aided engineering) driven durability model verification for the automotive structure development", Finite Elements in Analysis and Design, vol.45(5), pp.324-332, 2009.

[7] C. R. Lee, J. W. Kim and J. O. Hallquist, "Validation of a FEA Tire Model for Vehicle Dynamic Analysis and Full Vehicle Real Time Proving Ground Simulations", SAE971100

[8] G. Q. Guang and Y. Fang, "Time-Domain Simulation and Analysis of Vehicle Ride Comfort", Automobile Technology, vol.8(2), pp.8-11, 2007.

[9] Changchun Automobile Research Institute, "GB/T4970-1996 Method of random input running test-Automotive ride comfort", 1996.

[10] H. Zhao and S. F. Lu, "Road input in time domain model on four tires vehicle", Automotive Engineering, vol.21(2), pp.112-117, 1999.

[11] J. Yang, Y. Suematsu and Z. Kang, "Two-degree- offreedom controller to reduce the vibration of vehicle engine-body system", IEEE Transactions on Control Technology, vol.9(2), pp.295-317, 2001.

[12] R. A. Williams, "Automotive active suspensions", Part 1: basic principles, Proceeding of Institute of Mechanical Engineers, pp.415-426, 1997.

[13] Q. Zhu and M. C. Ishitobi, "Chaos and bifurcations in a nonlinear vehicle model", Journal of Sound and Vibration, vol.275(5), pp.1136-1146, 2004.

[14] M. Yamashita, K. Fujimofi, K. Hayakawa, et a1, "Application of $\mathrm{H}$ control to active suspension systems", Automatica, vol.30(11), pp.1717-1729, 1994.

[15] G. H. Tian, Y. S. Wang, A. L. Geng, "Time-domain linear simulation of automobile ride performance and software development", Machinery Design and Manufacture, vol.38(5), pp.80-82, 2006.

[16] W. Schiehlen and B. Hu, "Spectral simulation and shock absorber identification", International Journal of Non-Linear Mechanics, vol.38(2), pp.161-171, 2003.

[17] S. X. Gao, "Comment of Motor Vehicle Ride Vibration Evaluation", Automobile Technology, vol.12(3), pp.16-17, 1995. 